\title{
Continuous infusion of low-dose unfractionated heparin after aneurysmal subarachnoid hemorrhage: a preliminary study of cognitive outcomes
}

\author{
Robert F. James, MD,,2 Nicolas K. Khattar, MD, ${ }^{1}$ Zaid S. Aljuboori, MD, ${ }^{1}$ Paul S. Page, MD, ${ }^{1}$ \\ Elaine Y. Shao, MD, ${ }^{2}$ Lacey M. Carter, MD, ${ }^{2}$ Kimberly S. Meyer, PhD, ${ }^{1}$ Michael W. Daniels, MS, ${ }^{3}$ \\ John Craycroft, MS, ${ }^{3}$ John R. Gaughen Jr., MD, ${ }^{4}$ M. Imran Chaudry, MD, ${ }^{5}$ Shesh N. Rai, PhD, ${ }^{3}$ D. \\ Erik Everhart, $\mathrm{PhD},{ }^{6,7}$ and J. Marc Simard, MD, $\mathrm{PhD}^{8-10}$
}

\begin{abstract}
${ }^{1}$ Department of Neurological Surgery, University of Louisville School of Medicine, Louisville, Kentucky; 2 Department of Surgery, Brody School of Medicine at East Carolina University, Greenville, North Carolina; ' ${ }^{3}$ Department of Bioinformatics and Biostatistics, University of Louisville School of Public Health, Louisville, Kentucky; ${ }^{2}$ Sentara Martha Jefferson Hospital, Charlottesville, Virginia; ${ }^{5}$ Comprehensive Stroke and Cerebrovascular Center, Medical University of South Carolina, Charleston, South Carolina; Departments of ${ }^{6}$ Psychology and ${ }^{7}$ Internal Medicine, East Carolina University, Greenville, North Carolina; and Departments of ${ }^{8}$ Neurosurgery, ${ }^{9}$ Pathology, and ${ }^{10}$ Physiology, University of Maryland School of Medicine, Baltimore, Maryland
\end{abstract}

OBJECTIVE Cognitive dysfunction occurs in up to $70 \%$ of aneurysmal subarachnoid hemorrhage (aSAH) survivors. Low-dose intravenous heparin (LDIVH) infusion using the Maryland protocol was recently shown to reduce clinical vasospasm and vasospasm-related infarction. In this study, the Montreal Cognitive Assessment (MoCA) was used to evaluate cognitive changes in aSAH patients treated with the Maryland LDIVH protocol compared with controls.

METHODS A retrospective analysis of all patients treated for aSAH between July 2009 and April 2014 was conducted. Beginning in 2012, aSAH patients were treated with LDIVH in the postprocedural period. The MoCA was administered to all aSAH survivors prospectively during routine follow-up visits, at least 3 months after aSAH, by trained staff blinded to treatment status. Mean MoCA scores were compared between groups, and regression analyses were performed for relevant factors.

RESULTS No significant differences in baseline characteristics were observed between groups. The mean MoCA score for the LDIVH group $(n=25)$ was 26.4 compared with 22.7 in controls $(n=22)(p=0.013)$. Serious cognitive impairment (MoCA $\leq 20$ ) was observed in $32 \%$ of controls compared with $0 \%$ in the LDIVH group $(p=0.008)$. Linear regression analysis demonstrated that only LDIVH was associated with a positive influence on MoCA scores $(\beta=3.68, p=0.019)$, whereas anterior communicating artery aneurysms and fevers were negatively associated with MoCA scores. Multivariable linear regression analysis resulted in all 3 factors maintaining significance. There were no treatment complications.

CONCLUSIONS This preliminary study suggests that the Maryland LDIVH protocol may improve cognitive outcomes in aSAH patients. A randomized controlled trial is needed to determine the safety and potential benefit of unfractionated heparin in aSAH patients.

https://thejns.org/doi/abs/10.3171/2017.11.JNS17894

KEYWORDS low-dose intravenous heparin; subarachnoid hemorrhage; cognitive dysfunction; vascular disorders

A NEURYSMAL subarachnoid hemorrhage (aSAH) is a rare, devastating cause of stroke that carries a case fatality rate of up to $67 \% .{ }^{31}$ With improvements in aSAH treatment and ICU care, more patients who have experienced aSAH are surviving the initial event. Long- term cognitive impairment occurs in up to $70 \%$ of patients surviving the initial hemorrhage. ${ }^{1,28}$ Cognitive impairments often co-manifest with life-changing psychosocial problems, with $59 \%$ of surviving patients unable to return to their previous occupation, and up to $93 \%$ demonstrat-

ABBREVIATIONS ACOA = anterior communicating artery; aSAH = aneurysmal subarachnoid hemorrhage; DND = delayed neurological deficit; LDIVH = low-dose intravenous heparin; MMSE = Mini-Mental State Examination; MoCA = Montreal Cognitive Assessment; mRS = modified Rankin Scale; WFNS = World Federation of Neurological Societies.

SUBMITTED April 10, 2017. ACCEPTED November 29, 2017.

INCLUDE WHEN CITING Published online May 11, 2018; DOI: 10.3171/2017.11.JNS17894. 
ing deficits in activities of daily living.,18 Verbal memory, visual memory, language, and executive function are the most commonly impaired cognitive domains ${ }^{26}$ Although aSAH-related cognitive dysfunction may improve over time, most patients report lifelong cognitive deficits. ${ }^{14,24}$

Additional brain injury occurring after the initial hemorrhage may be due to cerebral vasospasm, inflammation, nonobstructive hydrocephalus, microthombi, cortical spreading depolarization, and neuronal cell death. ${ }^{6,25,36,37 \text {, }}$ 49,52,59 Specifically, microthrombi and intraparenchymal vasoconstriction have been associated with increased expression of membrane P-Selectin and decreased nitric oxide (NO), as well as ensuing adjacent neuronal apoptosis. ${ }^{39}$ Collectively, these pathologic processes result in delayed neurological deficits (DND). Cognitive dysfunction after aSAH is arguably one of the most critical manifestations of DND.

Subarachnoid hemorrhage induces a significant inflammatory response involving numerous cytokines, chemokines, complement, leukocytes, and microglia. ${ }^{15,22,35,46,48}$ The systemic inflammatory response has been demonstrated to correlate with cerebral vasospasm. Additionally, long-term cognitive dysfunction may also be significantly related to neuroinflammation. Inflammatory responses of the same sort result in similar patterns of cognitive dysfunction in diseases as varied as Alzheimer's disease, HIV, infection, traumatic brain injury, and normal aging. 12,19,21,38 Neuroimaging many years after aSAH frequently demonstrates findings of global cerebral atrophy, ventricular dilation, and sulcal enlargement, findings similar to those observed in other disease processes with cognitive dysfunction. ${ }^{2}$

Recent studies have shown the potential benefit of unfractionated heparin in antagonizing mechanisms responsible for DND associated with aSAH. ${ }^{44-46}$ Unfractionated heparin is a mixture of glycosaminoglycans of variable lengths and molecular weights ranging from 3 to 30 $\mathrm{kD} \cdot{ }^{23}$ As the most negatively charged biological molecule known, heparin has a strong ability to interfere with the functioning of positively charged molecules. Due to the difference in charges, heparin has been documented to interact with over 100 proteins. ${ }^{57}$ Interleukins, cytokines, and receptors located on endothelial cells, which are involved in the acute phase response, are positively charged, and thus are a reasonable target for the modulating effects of heparin. Heparin has strong antiinflammatory effects with many possible mechanisms, including binding to cell-surface glycosaminoglycans, preventing leukocyte migration, direct binding to chemokines and cytokines, and inhibition of intracellular NF- $\kappa B$. ${ }^{11,20,45,50,51}$ Heparin is also able to modulate endothelin-1 (ET-1) activity as well as scavenge free radicals, in addition to upregulation of superoxide dismutase. ${ }^{45,46}$ Significant vasoconstriction due to ET-1 is mediated in vascular smooth muscle cells through the epidermal growth factor receptor (EGFR). Heparin-binding epidermal growth factor, a ligand of EGFR, modulates its transactivation. ${ }^{4}$ Low-dose intravenous heparin (LDIVH) provides anticoagulation effects, which may counter the effects of aSAH-induced microthrombi, in addition to all the antiinflammatory effects of heparin. ${ }^{45}$ Preclinical studies have demonstrated that neuroinflammation after aSAH can be attenuated through the use of LDIVH, resulting in lessened tissue injury and decreased transsynaptic apoptosis. ${ }^{46}$

Given the report that unfractionated heparin reduces symptomatic cerebral vasospasm and vasospasm-related infarctions, we evaluated its effects on cognitive outcomes. The Montreal Cognitive Assessment (MoCA) is an alternative assessment to the Mini-Mental State Examination (MMSE) and, according to the National Institute of Neurological Disorders, is currently considered the gold standard for rapid cognitive screening in the setting of stroke. ${ }^{30}$ Studies have demonstrated that MoCA testing is more sensitive than MMSE in detecting cognitive dysfunction following aSAH. ${ }^{1,17,41,53}$ Given MoCA's emphasis on language, memory, and visuospatial functioning, it is a highly sensitive and effective assessment tool for identifying even mild cognitive impairment. ${ }^{41,58}$

In the present study, we evaluated cognitive outcomes in patients with aSAH treated with standard therapy compared with those treated with LDIVH.

\section{Methods}

With approval from the institutional review board of the East Carolina University Brody School of Medicine and Vidant Medical Center, we reviewed the records of all patients with aSAH treated by clipping or coiling by a single cerebrovascular neurosurgeon (R.F.J.) between July 2009 and April 2014. Baseline evaluations included CT, vascular imaging, and determination of Fisher grade and World Federation of Neurological Societies (WFNS) grade. All patients were treated according to the American Heart Association's Guidelines for the Management of Aneurysmal Subarachnoid Hemorrhage, ${ }^{5,8}$ with an emphasis on prompt aneurysm obliteration, drainage of cerebrospinal fluid for treatment of hydrocephalus, and maintenance of euvolemia. Patients were treated with $60 \mathrm{mg}$ of oral nimodipine every 4 hours beginning within 48 hours of the ictus, in accordance with the American Heart Association guidelines. ${ }^{5}$ Radiographic studies, such as CT, CT angiography (CTA), digital subtraction angiography (DSA), and/or MR angiography (MRA) were routinely performed within 24 hours of treatment to confirm obliteration of the aneurysm, rule out any complication of treatment, and evaluate for the development of hydrocephalus.

In 2012 we began routinely treating patients with secured aSAH with an LDIVH protocol as a new addition to our previous standard of care. The control group included patients receiving the standard of care treatment prior to implementation of the new LDIVH standard of care protocol. The treatment group included patients receiving the newly established LDIVH protocol in addition to the previous standard of care therapy. No patients prior to 2012 were treated with the LDIVH protocol. Concurrently in 2012, it became our standard of care to perform cognitive screening on aSAH patients at 90 days after hemorrhage or longer. Blinded nonstudy personnel, who were unaware of inpatient heparin treatment protocols, administered MoCA testing to all aSAH patients during routinely scheduled follow-up visits, at least 3 months after surgery. Of all aSAH patients treated between July 2009 
TABLE 1. Baseline patient characteristics

\begin{tabular}{|c|c|c|c|}
\hline Characteristics & Control $(n=22)$ & Heparin $(n=25)$ & p Value* \\
\hline Mean age in yrs ( \pm SEM) & $51.2 \pm 2.3$ & $50.9 \pm 2.5$ & 0.92 \\
\hline Fisher grade 3 & 8 & 14 & 0.24 \\
\hline WFNS grade & & & 0.66 \\
\hline I & 13 & 15 & \\
\hline II & 3 & 6 & \\
\hline III & 1 & 1 & \\
\hline IV & 3 & 3 & \\
\hline V & 2 & 0 & \\
\hline WNFS grade $\geq I V$ & 5 & 3 & 0.56 \\
\hline Female sex & 17 & 17 & 0.70 \\
\hline Anterior circulation aneurysm & 19 & 23 & 0.88 \\
\hline $\mathrm{ACoA}$ aneurysm & 10 & 5 & 0.120 \\
\hline Microsurgical clipping & 2 & 6 & 0.33 \\
\hline Days from ictus to admission (mean \pm SD) & $1.6 \pm 3.6$ & $0.8 \pm 2.0$ & 0.37 \\
\hline Premorbid disability (reported) & 2 & 1 & 0.91 \\
\hline History of dementia & 2 & 2 & $>0.99$ \\
\hline Hypertension & 13 & 19 & 0.35 \\
\hline Tobacco use & 15 & 14 & $>0.99$ \\
\hline Marijuana use & 1 & 7 & 0.081 \\
\hline Cocaine use & 1 & 3 & 0.70 \\
\hline Alcohol use & 5 & 8 & 0.70 \\
\hline Heroin use & 1 & 0 & 0.95 \\
\hline
\end{tabular}

and April 2014, only those patients who had routine cognitive screening with MoCA testing at least 90 days after the $\mathrm{SAH}$ were included in this analysis. We excluded patients from LDIVH treatment if they were deemed to be at a higher risk for hemorrhagic sequelae, namely, presence of a significant tract hemorrhage following ventriculostomy catheter placement; significant contusion following surgical clipping; angiogram-negative $\mathrm{SAH}$; fusiform, mycotic, or blister aneurysms; unsecured aneurysms; patients who had received heparin in the 100 days prior to admission; and delayed presentation.

In the treatment group, patients received LDIVH (10$12 \mathrm{U} / \mathrm{kg} / \mathrm{hr}$ for approximately 14 days; FDA off-label) beginning 12 hours after aneurysm treatment and continuing until day 14 following the ictus, with activated partial thromboplastin time routinely monitored to ensure that significant anticoagulation was not reached. Of a total of 47 aSAH patients included in the study during this time period, 22 received conventional management and had MoCA scores available, and 25 received LDIVH treatment and had MoCA scores available. In a few cases, more than one MoCA score was available, in which case the most recent score was used.

\section{Statistical Analysis}

Descriptive statistics such as frequency and proportion for any discrete variable and mean and standard deviation for any continuous variable are reported. Associations be- tween discrete groups were compared using a chi-square test or Fisher's exact test. Two-group means were compared using a 2-sample t-test. The mean MoCA scores were compared using a 1-tailed t-test for unequal variance. Statistical significance was determined as $p<0.05$. Linear regression analysis of MoCA scores was performed for multiple potential factors and predictors. Significant factors were simultaneously controlled for using a multivariable linear regression analysis. The data were analyzed using SAS statistical software (version 3.0, SAS Institute).

\section{Results}

A total of 47 patients with aSAH were included in the study: 22 patients were treated with standard therapy and 25 were treated with LDIVH. Baseline characteristics demonstrated no statistically significant differences in age, dementia history, sex, Fisher grade, WFNS grade, or number of anterior circulation aneurysms between the control and heparin-treated groups (Table 1).

The mean MoCA scores were significantly different between the groups at follow-up (Table 2). Those treated with LDIVH demonstrated a mean MoCA score of $26.4 \pm 2.3$ compared with $22.7 \pm 7.0$ in the control group $(\mathrm{p}=0.013$, Fig. 1). Serious cognitive impairment, defined by MoCA score $\leq 20$, did not occur in any of the patients treated with LDIVH, compared with 7 in the control group $(\mathrm{p}=0.008)$. The was no significant difference in modified Rankin 
TABLE 2. Results

\begin{tabular}{lccc}
\hline \multicolumn{1}{c}{ Outcome } & Control $(\mathrm{n}=22)$ & Heparin $(\mathrm{n}=25)$ & p Value* \\
\hline Mean MoCA score & $22.7 \pm 7.0$ & $26.4 \pm 2.3$ & $0.013 \dagger$ \\
\hline MoCA score $\leq 20$ & 7 & 0 & 0.008 \\
\hline Mos to follow-up MoCA testing & $25.4 \pm 12.6$ & $7.1 \pm 7.4$ & $<0.001$ \\
\hline Mean mRS score & $1.4 \pm 0.8$ & $1.1 \pm 0.8$ & 0.25 \\
\hline mRS score $\leq 1$ & 13 & 20 & 0.21 \\
\hline Mean length of hospital stay in days & $16.4 \pm 8.0$ & $16.2 \pm 5.2$ & 0.92 \\
\hline Days on continuous IV heparin drip & $\mathrm{NA}$ & $11.9 \pm 3.6$ & $\mathrm{NA}$ \\
\hline SQ heparin DVT prophylaxis & 11 & 11 & 0.91 \\
\hline Mean total units of heparin per patient & $98,636 \pm 43,880$ & $248,644 \pm 128,696$ & 1.0 \\
\hline Hydrocephalus requiring EVD & 7 & 8 & 0.169 \\
\hline Hydrocephalus requiring VPS & 6 & 4 & 1.0 \\
\hline Any fever episodes $\left(>101.6^{\circ} \mathrm{F}\right)$ & 4 & 4 & 0.79 \\
\hline Multiple fever episodes $\left(>101.6^{\circ} \mathrm{F}\right)$ & 2 & 5 & 0.81 \\
\hline Discharge to rehab or SNF & 6 & 2 & \\
\hline
\end{tabular}

DVT = deep venous thrombosis; $E V D$ = external ventricular drain; IV = intravenous; rehab = inpatient rehabilitation hospital; $N A$ = not applicable; SNF = skilled nursing facility; SQ = subcutaneous; VPS = ventriculoperitoneal shunt.

Values are presented as the number of patients unless stated otherwise.

* Unless stated otherwise, chi-square or Fisher's exact test, where appropriate for frequency comparisons; 2-tailed independent Student's t-test for continuous variables.

† One-tailed unequal variances t-test.

Scale (mRS) score between the groups, with a mean score of $1.4 \pm 0.8$ in the control group compared with $1.1 \pm 0.8$ in the LDIVH group $(p=0.25)$. Time from aneurysm rupture to MoCA assessment at follow-up was significantly different between the two groups $(\mathrm{p}<0.001)$. Patients treated with heparin had a mean time to follow-up of $7.1 \pm 7.4$ months compared with $25.4 \pm 12.6$ months in the control

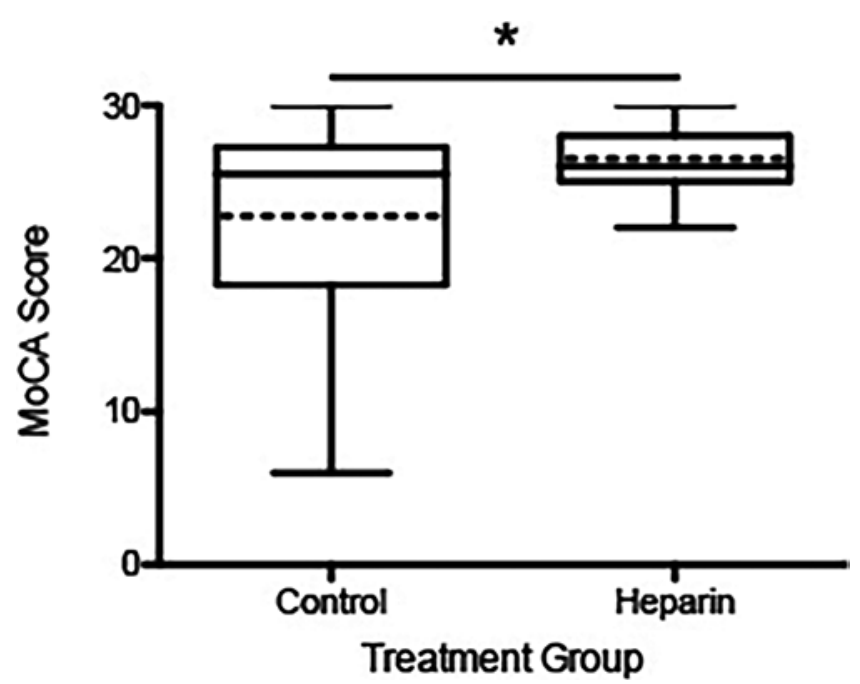

FIG. 1. Box-and-whiskers plot showing the distribution of MoCA scores for each treatment group. Note the tight packing and decreased variance of MoCA scores in the LDIVH treatment group. The solid horizontal line indicates the median; dashed horizontal line, mean; box, interquartile range; and whiskers, minimum to maximum. Asterisk denotes significance between the two groups $(p=0.013)$ in regard to a comparison of the mean. group. There were no hemorrhagic complications or any cases of type II heparin-induced thrombocytopenia.

Linear regression analysis of factors related to the distribution of MoCA scores demonstrated that only LDIVH treatment was associated with a positive influence on MoCA scores $(\beta=3.68, p=0.017$; Table 3$)$. Anterior communicating artery aneurysm location and multiple fevers were negatively associated with MoCA scores $(\beta=-4.85$, $\mathrm{p}=0.003[\mathrm{ACoA}] ; \beta=-6.27, \mathrm{p}=0.006$ [multiple fevers]). A Fisher grade of 3 , vasospasm-related infarct, and WFNS grade $\geq$ IV did not demonstrate significant correlation with poor MoCA score. Following multivariable linear regression analysis, LDIVH remained positively associated with improved MoCA scores ( $p=0.019)$; ACoA aneurysm location $(p=0.001)$ and fevers $(p=0.0001)$ also remained significant negative correlates with cognitive scores. No treatment complications occurred in either group. LDIVH treatment did not result in significant anticoagulation.

\section{Discussion}

Early treatment of aneurysms, the development of treatment protocols, and the development of neurocritical care as a unique specialty together have led to tremendous improvements in overall morbidity and mortality for aSAH patients. However, long-term cognitive dysfunction, often discovered in the months following aSAH, has emerged as a newly recognized delayed sequela. ${ }^{28,31,45,47}$ To date, no study has shown any treatment that improves cognitive outcome after aSAH. Our preliminary study suggests that the use of LDIVH prophylaxis, in addition to standard therapy, may safely reduce the incidence of cognitive dysfunction experienced by patients after aSAH compared with those treated with standard therapy alone. Our study builds on a previous study by Simard et al.,44 which provided self- 
TABLE 3. Linear regression for factors related to the distribution of MoCA scores

\begin{tabular}{|c|c|c|c|c|c|}
\hline Factor & $\beta$ & $\beta$ (SE) & Univariate $p$ Value & $\mathrm{R}^{2}$ & Multivariable $p$ Value \\
\hline Male sex & 2.90 & 1.71 & 0.097 & 0.0601 & \\
\hline Heparin treatment group & 3.68 & 1.49 & 0.017 & 0.1005 & 0.019 \\
\hline Imaging vasospasm & -0.11 & 0.95 & 0.91 & 0.0044 & \\
\hline Educational level >12 yrs & 3.64 & 2.64 & 0.183 & 0.0870 & \\
\hline Fisher grade 3 & 1.11 & 1.57 & 0.49 & 0.0109 & \\
\hline Vasospasm-related infarct & -3.03 & 3.18 & 0.35 & 0.0260 & \\
\hline WFNS grade $\geq I V$ & -1.93 & 2.20 & 0.39 & 0.0167 & \\
\hline Hypertension & -1.80 & 1.67 & 0.29 & 0.0252 & \\
\hline Premorbid dysfunction & 2.17 & 3.21 & 0.50 & 0.0100 & \\
\hline Discharge to rehab & -3.44 & 1.79 & 0.061 & 0.0758 & \\
\hline Coiling treatment & -0.74 & 2.10 & 0.73 & 0.0027 & \\
\hline Posterior circulation aneurysm & 2.64 & 2.53 & 0.31 & 0.0237 & \\
\hline ACoA aneurysm & -4.85 & 1.53 & 0.003 & 0.1825 & 0.001 \\
\hline Ventricular drainage & -1.13 & 1.69 & 0.50 & 0.0099 & \\
\hline CSF shunt procedure & 0.59 & 2.10 & 0.78 & 0.0017 & \\
\hline Chemical DVT prophylaxis & 0.94 & 1.58 & 0.55 & 0.0078 & \\
\hline Any fever $\left(>101.6^{\circ} \mathrm{F}\right)^{*}$ & -6.04 & 1.90 & 0.003 & 0.1837 & \\
\hline Multiple fevers & -6.27 & 2.17 & 0.006 & 0.1561 & $<0.001$ \\
\hline Isolated fever & -3.80 & 3.87 & 0.33 & 0.0210 & \\
\hline Levetiracetam & -1.81 & 3.22 & 0.58 & 0.0070 & \\
\hline \multicolumn{6}{|l|}{ Continuous predictors } \\
\hline Age & -0.03 & 0.07 & 0.64 & 0.0049 & \\
\hline Length of stay & -0.17 & 0.12 & 0.80 & 0.0014 & \\
\hline
\end{tabular}

SE = standard error.

When $\beta$ is positive and statistically significant, the factor can be considered to positively influence MoCA score distribution. The reverse is true when $\beta$ is negative and statistically significant. Only those factors with significant univariate $p$ values $(<0.05)$ were selected for multivariable linear regression analysis.

* "Any fever" was not selected as a factor for multivariable linear regression, as this is a combination of 2 component factors ("multiple fevers" and "isolated fever").

Rather, these component fever factors were considered separately for multivariable linear regression.

reported cognitive outcomes rather than formal cognitive testing. Two previous randomized trials evaluated lowmolecular-weight heparin in aSAH with conflicting results regarding vasospasm, ${ }^{43,56}$ although they did not evaluate cognitive outcomes. The current study and the study by Simard et al. differ from the aforementioned studies in that a continuous infusion of unfractionated heparin was administered, rather than scheduled subcutaneous injections of low-molecular-weight heparin. It is possible that either the route or the schedule of administration or the type of heparin may influence results. ${ }^{43,56}$

Both the occurrence and the severity of fever have been well documented as negative predictive factors of neurological outcomes in aSAH. ${ }^{13,33}$ One prospective study evaluating 353 patients demonstrated that patients with an mRS score of 4-6 demonstrated a mean maximum temperature of $0.7^{\circ} \mathrm{C}$ higher than those with an mRS score of $0-3 .^{13}$ While the etiology of thermoregulatory dysfunction is based on animal models after acute brain injury, it is believed to be a result of delayed neurological insults affecting the hypothalamus, midbrain, or pons. ${ }^{16,42}$ In our study, fever was negatively correlated with worse MoCA scores in both treatment groups, consistent with prior reports. ${ }^{54}$

ACoA aneurysms are a significant independent risk factor for the development of cognitive dysfunction after
aSAH. ${ }^{3,26}$ Kreiter et al. evaluated 113 patients with ACoA aneurysms with neuropsychological testing at a mean interval of $107 \pm 21$ days. The results demonstrated that nonposterior circulation aneurysms were independent predictors of abnormal visual memory and verbal memory. ${ }^{26}$ More recently, a significant number of studies evaluated the incidence of adverse cognitive outcomes in patients with ACoA aneurysms compared with other locations and were unable to demonstrate this correlation. . $^{10,27,32,40}$ Manning et al. showed that patients with ACoA aneurysms performed better on the Tower of London executive function test than patients whose ruptured aneurysm was in other locations ${ }^{27}$ It is important to note that there was significant variability in the time to follow-up examination, which can easily confound correlations. This is evident in the study by Ogden et al., where patient follow-up evaluations ranged from 7 to 115 months after craniotomy. ${ }^{32}$ Variability in the testing could potentially mask true differences in cognition between the various aneurysm locations due to the possibility of natural temporal improvement. In our study, there were 10 patients with ACoA aneurysms in the control group and 5 in the LDIVH treatment group $(\mathrm{p}=$ 0.12 ), and ACoA aneurysm location was one of the factors that emerged in our multivariable linear regression analysis. 
Cognitive dysfunction is present in up to $70 \%$ of patients after aSAH. ${ }^{28,47}$ While prior studies have evaluated aSAH outcomes using the mRS, this functional test lacks the sensitivity necessary to evaluate cognitive outcomes, compared with MoCA testing. Significant clinical and radiological similarities exist between the cognitive effects of aSAH and those seen in Alzheimer's disease, Parkinson's disease, depression, and normal aging. ${ }^{2,34}$ The MoCA scale is effective in evaluating all of these conditions, making it an excellent test for the evaluation of cognitive outcomes after aSAH. ${ }^{7,30}$ While both the MMSE and MoCA test memory and language, the MMSE does not adequately evaluate executive function, which has been documented to be abnormal in up to $76 \%$ of aSAH patients. ${ }^{1}$ MoCA places significant emphasis on the evaluation of frontal lobe function such as attention as well as executive function, and has been studied extensively in the setting of poststroke cognitive impairment. ${ }^{9,29}$ A recent prospective study in 80 patients with aSAH compared results obtained using both MMSE and MoCA and showed a greater area under the receiver operating characteristic curve for MoCA (0.92 vs $0.77, p=0.009){ }^{1,55}$ MoCA testing can be completed in approximately 20 minutes in an alert patient with multiple cognitive domains tested, similar to those evaluated in formal, multihour, neuropsychological testing. MoCA is therefore a rapid and accurate tool for evaluating the effects of aSAH on multiple cognitive domains.

The present study has significant limitations, including its nonrandomized retrospective design and modest sample size. One potential limitation of the study includes the imbalance between the number of patients presenting with ACoA aneurysms in the treatment and control groups, with a greater proportion found in the control group. ACoA aneurysms are generally associated with more severe longterm cognitive deficits, and we did find a similar association between ACoA aneurysm location and decreased MoCA score distribution in our study. While the increased frequency of ACoA aneurysms in the control group appears to have contributed partially to this group's poor MoCA score distribution, our multivariate analysis establishes that when controlling for this effect of ACoA aneurysm location, LDIVH treatment remained a significant positive influence on MoCA score distribution for patients with and without ruptured ACoA aneurysms. The number of patients who underwent surgical clipping included in either cohort is also potentially imbalanced with a larger proportion found within the LDIVH treatment group. Current scientific opinion considers that surgical clipping may have a greater negative effect on cognitive outcomes than endovascular treatment. If this effect is true, the imbalance found between our groups would be expected to influence poorer cognitive outcomes in the LDIVH treatment group and would decrease the chances of our study demonstrating a positive treatment effect of LDIVH on cognition, whereas we found the opposite. Additionally, in our univariate model, aneurysm treatment method was not found to be significantly associated with MoCA score distribution. Another limitation includes the time at which the MoCA was administered. Patients treated with standard therapy had a significantly longer time to MoCA testing (25.4 \pm 12.6 months) compared with those treated with
LDIVH (7.1 \pm 7.4 months, $\mathrm{p}<0.001)$. Longer duration to examination usually correlates with improvement in cognitive outcomes and thus would favor improvement in patient outcomes in the control group, which was not observed. It could be postulated that this timing difference may have masked a greater treatment effect than what was seen in our data. Improvement in physician procedural skills and progression on the learning curve also may have contributed to an improvement in outcomes.

This study is the second to demonstrate the potential safety and efficacy of the Maryland LDIVH protocol in the prevention of DND in aSAH patients. Our results provide a compelling rationale for a randomized controlled trial to evaluate LDIVH's safety and potential efficacy in aSAH. ${ }^{44,45}$ The Aneurysmal Subarachnoid Hemorrhage Trial Randomizing Heparin (ASTROH) is a phase 2 randomized multicenter trial currently enrolling subjects to evaluate LDIVH in aSAH patients (clinical trial registration no. NCT02501434, clinicaltrials.gov).

\section{Conclusions}

Heparin is a potent antiinflammatory agent that has been correlated with a reduction in neuroinflammation associated with aSAH. ${ }^{46}$ Previous studies have demonstrated LDIVH provides protection from vasospasm and may have a positive effect on memory and cognitive outcomes..$^{21,44}$ The present study suggests that the Maryland LDIVH protocol is safe and may reduce poor cognitive outcomes in patients treated after aSAH. Our results support continued enrollment efforts in ASTROH, a phase 2 randomized trial evaluating the safety and possible efficacy of LDIVH in aSAH.

\section{Acknowledgments}

Dr. Rai is supported in part by Wendell Cherry Chair in Clinical Trial Research funds and Dr. D. M. Miller, Director, James Graham Brown Cancer Center. Dr. Simard is supported in part by grants from NINDS (NS060801, NS102589), NIBIB (EB015870), and the Veterans Administration (BX001629, BX002889). Dr. James is supported in part by a grant from the Brain Aneurysm Foundation. We thank Ryan G. Nazar, MD, and Darlene A. Burke, MS, for their help with data analysis.

\section{References}

1. Al-Khindi T, Macdonald RL, Schweizer TA: Cognitive and functional outcome after aneurysmal subarachnoid hemorrhage. Stroke 41:e519-e536, 2010

2. Bendel P, Koivisto T, Aikiä M, Niskanen E, Könönen M, Hänninen T, et al: Atrophic enlargement of CSF volume after subarachnoid hemorrhage: correlation with neuropsychological outcome. AJNR Am J Neuroradiol 31:370-376, 2010

3. Bornstein RA, Weir BK, Petruk KC, Disney LB: Neuropsychological function in patients after subarachnoid hemorrhage. Neurosurgery 21:651-654, 1987

4. Chansel D, Ciroldi M, Vandermeersch S, Jackson LF, Gomez AM, Henrion D, et al: Heparin binding EGF is necessary for vasospastic response to endothelin. FASEB J 20:1936-1938, 2006

5. Connolly ES Jr, Rabinstein AA, Carhuapoma JR, Derdeyn CP, Dion J, Higashida RT, et al: Guidelines for the management of aneurysmal subarachnoid hemorrhage: a guideline for healthcare professionals from the American Heart Asso- 
ciation/American Stroke Association. Stroke 43:1711-1737, 2012

6. Crowley RW, Medel R, Kassell NF, Dumont AS: New insights into the causes and therapy of cerebral vasospasm following subarachnoid hemorrhage. Drug Discov Today 13:254-260, 2008

7. Dalrymple-Alford JC, MacAskill MR, Nakas CT, Livingston L, Graham C, Crucian GP, et al: The MoCA: well-suited screen for cognitive impairment in Parkinson disease. Neurology 75:1717-1725, 2010

8. Diringer MN, Bleck TP, Hemphill JC III, Menon D, Shutter L, Vespa P, et al: Critical care management of patients following aneurysmal subarachnoid hemorrhage: recommendations from the Neurocritical Care Society's Multidisciplinary Consensus Conference. Neurocrit Care 15:211-240, 2011

9. Dong Y, Venketasubramanian N, Chan BP, Sharma VK, Slavin MJ, Collinson SL, et al: Brief screening tests during acute admission in patients with mild stroke are predictive of vascular cognitive impairment 3-6 months after stroke. J Neurol Neurosurg Psychiatry 83:580-585, 2012

10. Egge A, Waterloo K, Sjøholm H, Ingebrigtsen T, Forsdahl $\mathrm{S}$, Jacobsen EA, et al: Outcome 1 year after aneurysmal subarachnoid hemorrhage: relation between cognitive performance and neuroimaging. Acta Neurol Scand 112:76-80, 2005

11. Elsayed E, Becker RC: The impact of heparin compounds on cellular inflammatory responses: a construct for future investigation and pharmaceutical development. J Thromb Thrombolysis 15:11-18, 2003

12. Faden AI, Wu J, Stoica BA, Loane DJ: Progressive inflammatory-mediated neurodegeneration after traumatic brain or spinal cord injury. Br J Pharmacol 173:681-691, 2016

13. Fernandez A, Schmidt JM, Claassen J, Pavlicova M, Huddleston D, Kreiter KT, et al: Fever after subarachnoid hemorrhage: risk factors and impact on outcome. Neurology 68:1013-1019, 2007

14. Frazer D, Ahuja A, Watkins L, Cipolotti L: Coiling versus clipping for the treatment of aneurysmal subarachnoid hemorrhage: a longitudinal investigation into cognitive outcome. Neurosurgery 60:434-442, 2007

15. Friedrich V, Flores R, Muller A, Bi W, Peerschke EI, Sehba FA: Reduction of neutrophil activity decreases early microvascular injury after subarachnoid haemorrhage. J Neuroinflammation 8:103, 2011

16. Frosini M, Sesti C, Valoti M, Palmi M, Fusi F, Parente L, et al: Rectal temperature and prostaglandin E2 increase in cerebrospinal fluid of conscious rabbits after intracerebroventricular injection of hemoglobin. Exp Brain Res 126:252-258, 1999

17. Godefroy O, Fickl A, Roussel M, Auribault C, Bugnicourt JM, Lamy C, et al: Is the Montreal Cognitive Assessment superior to the Mini-Mental State Examination to detect poststroke cognitive impairment? A study with neuropsychological evaluation. Stroke 42:1712-1716, 2011

18. Hellawell DJ, Taylor R, Pentland B: Persisting symptoms and carers' views of outcome after subarachnoid haemorrhage. Clin Rehabil 13:333-340, 1999

19. Heppner FL, Ransohoff RM, Becher B: Immune attack: the role of inflammation in Alzheimer disease. Nat Rev Neurosci 16:358-372, 2015

20. Hochart H, Jenkins PV, Smith OP, White B: Low-molecular weight and unfractionated heparins induce a downregulation of inflammation: decreased levels of proinflammatory cytokines and nuclear factor- $\kappa \mathrm{B}$ in LPS-stimulated human monocytes. Br J Haematol 133:62-67, 2006

21. Hong S, Banks WA: Role of the immune system in HIV-associated neuroinflammation and neurocognitive implications. Brain Behav Immun 45:1-12, 2015

22. Ishikawa M, Kusaka G, Yamaguchi N, Sekizuka E, Nakadate
$\mathrm{H}$, Minamitani $\mathrm{H}$, et al: Platelet and leukocyte adhesion in the microvasculature at the cerebral surface immediately after subarachnoid hemorrhage. Neurosurgery 64:546-554, 2009

23. Kandrotas RJ: Heparin pharmacokinetics and pharmacodynamics. Clin Pharmacokinet 22:359-374, 1992

24. Koivisto T, Vanninen R, Hurskainen H, Saari T, Hernesniemi J, Vapalahti M: Outcomes of early endovascular versus surgical treatment of ruptured cerebral aneurysms. A prospective randomized study. Stroke 31:2369-2377, 2000

25. Kranc KR, Pyne GJ, Tao L, Claridge TD, Harris DA, Cadoux-Hudson TA, et al: Oxidative degradation of bilirubin produces vasoactive compounds. Eur J Biochem 267:70947101,2000

26. Kreiter KT, Copeland D, Bernardini GL, Bates JE, Peery S, Claassen J, et al: Predictors of cognitive dysfunction after subarachnoid hemorrhage. Stroke 33:200-208, 2002

27. Manning L, Pierot L, Dufour A: Anterior and non-anterior ruptured aneurysms: memory and frontal lobe function performance following coiling. Eur J Neurol 12:466-474, 2005

28. Mavaddat N, Sahakian BJ, Hutchinson PJ, Kirkpatrick PJ: Cognition following subarachnoid hemorrhage from anterior communicating artery aneurysm: relation to timing of surgery. J Neurosurg 91:402-407, 1999

29. Mayer SA, Kreiter KT, Copeland D, Bernardini GL, Bates JE, Peery S, et al: Global and domain-specific cognitive impairment and outcome after subarachnoid hemorrhage. Neurology 59:1750-1758, 2002

30. Nasreddine ZS, Phillips NA, Bédirian V, Charbonneau S, Whitehead V, Collin I, et al: The Montreal Cognitive Assessment, MoCA: a brief screening tool for mild cognitive impairment. J Am Geriatr Soc 53:695-699, 2005

31. Nieuwkamp DJ, Setz LE, Algra A, Linn FH, de Rooij NK, Rinkel GJ: Changes in case fatality of aneurysmal subarachnoid haemorrhage over time, according to age, sex, and region: a meta-analysis. Lancet Neurol 8:635-642, 2009

32. Ogden JA, Mee EW, Henning M: A prospective study of impairment of cognition and memory and recovery after subarachnoid hemorrhage. Neurosurgery 33:572-587, 1993

33. Oliveira-Filho J, Ezzeddine MA, Segal AZ, Buonanno FS, Chang Y, Ogilvy CS, et al: Fever in subarachnoid hemorrhage: relationship to vasospasm and outcome. Neurology 56:1299-1304, 2001

34. Østergaard L, Aamand R, Karabegovic S, Tietze A, Blicher JU, Mikkelsen IK, et al: The role of the microcirculation in delayed cerebral ischemia and chronic degenerative changes after subarachnoid hemorrhage. J Cereb Blood Flow Metab 33:1825-1837, 2013

35. Provencio JJ, Fu X, Siu A, Rasmussen PA, Hazen SL, Ransohoff RM: CSF neutrophils are implicated in the development of vasospasm in subarachnoid hemorrhage. Neurocrit Care 12:244-251, 2010

36. Provencio JJ, Vora N: Subarachnoid hemorrhage and inflammation: bench to bedside and back. Semin Neurol 25:435444, 2005

37. Pyne-Geithman GJ, Caudell DN, Prakash P, Clark JF: Glutathione peroxidase and subarachnoid hemorrhage: implications for the role of oxidative stress in cerebral vasospasm. Neurol Res 31:195-199, 2009

38. Rosi S, Ramirez-Amaya V, Vazdarjanova A, Worley PF, Barnes CA, Wenk GL: Neuroinflammation alters the hippocampal pattern of behaviorally induced Arc expression. J Neurosci 25:723-731, 2005

39. Sabri M, Ai J, Lakovic K, D’abbondanza J, Ilodigwe D, Macdonald RL: Mechanisms of microthrombi formation after experimental subarachnoid hemorrhage. Neuroscience 224:26-37, 2012

40. Samra SK, Giordani B, Caveney AF, Clarke WR, Scott PA, Anderson S, et al: Recovery of cognitive function after 
surgery for aneurysmal subarachnoid hemorrhage. Stroke 38:1864-1872, 2007

41. Schweizer TA, Al-Khindi T, Macdonald RL: Mini-Mental State Examination versus Montreal Cognitive Assessment: rapid assessment tools for cognitive and functional outcome after aneurysmal subarachnoid hemorrhage. J Neurol Sci 316:137-140, 2012

42. Shibata M: Hyperthermia in brain hemorrhage. Med Hypotheses 50:185-190, 1998

43. Siironen J, Juvela S, Varis J, Porras M, Poussa K, Ilveskero S, et al: No effect of enoxaparin on outcome of aneurysmal subarachnoid hemorrhage: a randomized, double-blind, placebocontrolled clinical trial. J Neurosurg 99:953-959, 2003

44. Simard JM, Aldrich EF, Schreibman D, James RF, Polifka A, Beaty N: Low-dose intravenous heparin infusion in patients with aneurysmal subarachnoid hemorrhage: a preliminary assessment. J Neurosurg 119:1611-1619, 2013

45. Simard JM, Schreibman D, Aldrich EF, Stallmeyer B, Le B, James RF, et al: Unfractionated heparin: multitargeted therapy for delayed neurological deficits induced by subarachnoid hemorrhage. Neurocrit Care 13:439-449, 2010

46. Simard JM, Tosun C, Ivanova S, Kurland DB, Hong C, Radecki L, et al: Heparin reduces neuroinflammation and transsynaptic neuronal apoptosis in a model of subarachnoid hemorrhage. Transl Stroke Res 3 (Suppl 1):155-165, 2012

47. Sobey CG, Faraci FM: Subarachnoid haemorrhage: what happens to the cerebral arteries? Clin Exp Pharmacol Physiol 25:867-876, 1998

48. Sozen T, Tsuchiyama R, Hasegawa Y, Suzuki H, Jadhav V, Nishizawa $\mathrm{S}$, et al: Immunological response in early brain injury after SAH. Acta Neurochir Suppl 110:57-61, 2011

49. Stein SC, Levine JM, Nagpal S, LeRoux PD: Vasospasm as the sole cause of cerebral ischemia: how strong is the evidence? Neurosurg Focus 21(3):E2, 2006

50. Tyrrell DJ, Horne AP, Holme KR, Preuss JM, Page CP: Heparin in inflammation: potential therapeutic applications beyond anticoagulation. Adv Pharmacol 46:151-208, 1999

51. Tyrell DJ, Kilfeather S, Page CP: Therapeutic uses of heparin beyond its traditional role as an anticoagulant. Trends Pharmacol Sci 16:198-204, 1995

52. Vergouwen MD, Vermeulen M, Coert BA, Stroes ES, Roos YB: Microthrombosis after aneurysmal subarachnoid hemorrhage: an additional explanation for delayed cerebral ischemia. J Cereb Blood Flow Metab 28:1761-1770, 2008

53. Wong GK, Lam S, Ngai K, Wong A, Mok V, Poon WS: Evaluation of cognitive impairment by the Montreal cognitive assessment in patients with aneurysmal subarachnoid haemorrhage: prevalence, risk factors and correlations with 3 month outcomes. J Neurol Neurosurg Psychiatry 83:1112-1117, 2012

54. Wong GK, Lam SW, Ngai K, Wong A, Siu D, Poon WS, et al: Cognitive domain deficits in patients with aneurysmal subarachnoid haemorrhage at 1 year. J Neurol Neurosurg Psychiatry 84:1054-1058, 2013

55. Wong GK, Lam SW, Wong A, Ngai K, Poon WS, Mok V: Comparison of Montreal Cognitive Assessment and MiniMental State Examination in evaluating cognitive domain deficit following aneurysmal subarachnoid haemorrhage. PLoS One 8:e59946, 2013

56. Wurm G, Tomancok B, Nussbaumer K, Adelwöhrer C, Holl $\mathrm{K}$ : Reduction of ischemic sequelae following spontaneous subarachnoid hemorrhage: a double-blind, randomized comparison of enoxaparin versus placebo. Clin Neurol Neurosurg 106:97-103, 2004
57. Young E: The anti-inflammatory effects of heparin and related compounds. Thromb Res 122:743-752, 2008

58. Zadikoff C, Fox SH, Tang-Wai DF, Thomsen T, de Bie RM, Wadia P, et al: A comparison of the mini mental state exam to the Montreal cognitive assessment in identifying cognitive deficits in Parkinson's disease. Mov Disord 23:297-299, 2008

59. Zhang ZD, Macdonald RL: Contribution of the remodeling response to cerebral vasospasm. Neurol Res 28:713-720, 2006

\section{Disclosures}

The authors report the following. Dr. James: consultant for Medtronic and Pulsar Vascular (acquired by Johnson and Johnson, Inc.); investigator-initiated research grant funding from Medtronic, MicroVention, and Penumbra; shareholder, Remedy Pharmaceuticals; primary principal investigator for ASTROH Trial and PREDICT. Dr. Simard: co-principal investigator for ASTROH Trial; shareholder and Board of Directors, Remedy Pharmaceuticals. Dr. Chaudry: consultant for Penumbra, Medtronic, MicroVention, Pulsar Vascular (acquired by Johnson and Johnson, Inc.), Codman, and Blockade; shareholder, Three Rivers and Penumbra.

\section{Author Contributions}

Conception and design: James, Simard. Acquisition of data: James, Shao, Carter. Analysis and interpretation of data: James, Khattar, Aljuboori, Page, Shao, Carter, Daniels, Craycroft, Gaughen, Chaudry, Rai, Everhart, Simard. Drafting the article: James, Khattar, Aljuboori, Page, Shao, Carter. Critically revising the article: James, Khattar, Aljuboori, Page, Meyer, Rai, Everhart, Simard. Reviewed submitted version of manuscript: all authors. Statistical analysis: James, Daniels, Craycroft, Rai. Study supervision: James.

\section{Supplemental Information \\ Previous Presentations}

Portions of this work were presented at the AHA/ASA International Stroke Conference 2015, Nashville, Tennessee, February 11, 2015; the 14th Annual Neurocritical Care Society Meeting, National Harbor, Maryland, September 15-18, 2016; the 4th SNIS International Endovascular Stroke Conference (IESC) and 2015 AANS/CNS Joint Cerebrovascular Section Annual Meeting, Nashville, Tennessee, February 9-10, 2015; and the 82nd AANS Annual Scientific Meeting, San Francisco, California, April 5-9, 2014.

\section{Current Affiliations}

Paul S. Page: Department of Neurological Surgery, University of Wisconsin School of Medicine and Public Health, Madison, WI. Elaine Y. Shao: John T. Milliken Department of Medicine, Washington University School of Medicine, St Louis, MO.

Lacey B. Carter: Department of Neurosurgery, College of Medicine, The University of Oklahoma, Oklahoma City, OK.

\section{Correspondence}

Robert F. James: University of Louisville School of Medicine, Louisville, KY. robert.james@louisville.edu. 